\title{
Grey Relational Analysis of Obesity Measurements for Children
}

\section{Zheng $\mathrm{YB}^{1,2}$, Guo $\mathrm{HT}^{1,3}$, Zheng $\mathrm{XY}^{3}$, Xie $\mathrm{XF}^{1}$, Zeng $\mathrm{XS}^{1}$, Chen $\mathrm{SR}^{* 3}$ and $\mathrm{Li} \mathrm{LP}^{4}$}

${ }^{1}$ Shantou University Medical College (SUMC), Shantou, China

${ }^{2}$ Yue Bei people's hospital, Shaoguan, China

${ }^{3}$ Department of Endocrinology, the $2^{\text {nd }}$ Affiliated Hospital of Shantou University Medical College, Shantou, China

${ }^{4}$ Injury Prevention Research Center of Shantou University Medical College, Shantou, China

*Corresponding author: Chen SR, Department of Endocrinology, the $2^{\text {nd }}$ Affiliated Hospital of Shantou University Medical College, Shantou, Guangdong Province 515041, China, Fax: (0754) 88346543, Tel: (0754) 88915836, E-mail: chen-shenren@163.com

Citation: Zheng YB, Guo HT, Zheng XY, Xie XF, Zeng XS, et al. (2015) Grey Relational Analysis of Obesity Measurements for Children. J Obes Overweig 1(1): 105. doi: 10.15744/2455-7633.1.105

Received Date: February 27, 2015 Accepted Date: May 26, 2015 Published Date: May 28, 2015

\begin{abstract}
Objectives: To evaluate the relationship between body mass index (BMI) and various measurements such as waist circumference (WC), hip circumference (HC), waist-to-hip ratio (WHR), and waist-to-height ratio (WHtR) in obese children.

Design: Cross-sectional study.

Methods: A total of 512 obese children were identified in this study. And the data was analyzed using Grey Relational Analysis (GRA).

Results: For the males, the BMI scores were strongly correlated with the HC for children younger than 14 years, and this correlation was weaker for children older than 14 years. In addition, the BMI scores were closely associated with the WHtR and WC, but were weakly associated with WHR. For the females, however, distinct correlation regularities were not observed among the BMI, WC, HC, WHR, and WHtR.

Conclusion: The associations of the BMI scores with the WHtR and WC indicated that obesity mainly presents as abdominal obesity in children. HC, WHtR, and WC may be used as alternative parameters for measuring obesity, with considerations of age and gender. Keywords: Adolescent; Child; Grey relational analysis; Measurement; Obesity

Abbreviations: BMI: Body mass index; WC: Waist circumference; HC: Hip circumference; WHR: Waist-to-hip ratio; WHtR: Waistto-height ratio; GRA: Grey Relational Analysis; BF\%: Body fat percentage; CDC: Centers for Disease Control and Prevention; GRO: Grey relational order; GRG: Grey relational grade
\end{abstract}

\section{Introduction}

In recent years, the prevalence of obesity has been increasing in children. Many risk factors for overweight and obesity have been identified, including over-nutrition and lack of physical activities. People with obesity tend to develop chronic diseases such as cardiovascular diseases, diabetes mellitus, hypertension, dyslipidemia, and renal diseases [1-4]. Body mass index (BMI) is still one of the most important measurements and an essential indicator for evaluating nutritional status. However it has been found that BMI alone cannot indicate obesity comorbidities risk and mortality appropriately [5]. Over the past few decades, researchers have been making great efforts to find better measurements for obesity and its complications. BMI is calculated from weight and height, and it has been widely used and assumed to represent the body habitus. However, due to the differences in body composition, the BMI is no longer an accurate indicator of nutritional status [5]. The preferred and more accurate obesity metric in academic community is the body fat percentage $(\mathrm{BF} \%)$ - the ratio of the total weight of a person's fat to his or her body weight. Unfortunately, accurate measurement of the body fat percentage is much more difficult than measurement of BMI [6].Thus, many researches recommend other obesity measurements including waist circumference (WC), hip circumference (HC), waist-to-hip ratio (WHR), waist-to-height ratio (WHtR) and skinfold thickness [5,7]. They are related to fat distribution that takes account of the differences in body structure [5]. Children experience rapid physical growth and sexual maturity therefore the BMI scores of children are often categorized using age and gender. Many studies have been performed on the association between obesity and WC, HC, WHR, and WHtR [8-10]. However, WC and WHR usually have fixed cut-off values for adults, but not for children [11]. Therefore, in this research we aimed to evaluate the relationship between BMI and a variety of measurements such as WC, HC, WHR, and WHtR in obese children.

The problem on uncertainty existing commonly in nature and thinking, the ones on uncertainty in less data little sample, incomplete information and devoid of experience, which is just suitable to be dealt with by grey system theory [12]. The Grey Relational Analysis (GRA) aim at analyzing the relationships in behavior, posture and boundary (factor bounds, data bounds, relationship 
bounds, time bounds, and so on) [12]. It is obvious that GRA is characterized by (1) less data required (the needed data for GRA is as few as 3 in every series), (2) leaving out of consideration of data distribution, and (3) simple calculation [12]. For the limited sample size, we used GRA to explore the relationships between different obesity measurements in children.

\section{Subjects and Methods}

\section{Sampling and population}

A population-based study was conducted in Shantou, Guangdong Province, China, from June 2008 to June 2009. A total of 5,662 students, including 2,800 boys and 2,862 girls aged 6-18 years, were recruited for this study. Among them, 511 obese children aged 7-18 years were identified. Obese children aged 6 years or lesser were not found.

\section{Informed consent}

All the physical examination was completed after the signature of informed consent obtained from parents of all participants. The study protocol conformed to the ethical guidelines of the 1975 Declaration of Helsinki as reflected in a priori approval by the review committee of Shantou University Medical College. The study was approved by the Shantou Ethics Review Committee.

\section{Anthropometric measurements}

To ensure privacy, these students were individually examined in a private room. All the measurements were obtained twice and recorded to the nearest $0.01 \mathrm{~cm}$, using standard techniques and equipment. Measured for a third time if the first two differed and take the average of the three numbers.

Height and weight were measured while the participants were standing up straight, wore light clothing, and did not wear shoes. BMI was calculated as weight $(\mathrm{kg}) /$ height ${ }^{2}\left(\mathrm{~m}^{2}\right)$. Obese children were identified and included as subjects on the basis of the BMIfor-age growth charts [13] set by the Centers for Disease Control and Prevention (CDC).

Participants stood up straight and exhaled slowly. Removed any layers of clothing blocking waist and hip before measurement.. The WC was measured from midway between the top of the hipbone and the bottom of the rib cage. Held the end of the tape measure at the navel and brought it around the waist to the front. The measuring tape was parallel to the floor and fitted snugly around the torso without digging into the skin. After identifying the widest part of the hip, placed the tape measure at this location and measured around the circumference of the hip to get HC. The WHR and WHtR were calculated as WC $(\mathrm{cm}) / \mathrm{HC}(\mathrm{cm})$ and as WC $(\mathrm{cm}) /$ height $(\mathrm{cm})$, respectively.

\section{Quality control and statistical analysis}

All the data were entered into Microsoft Excel sheets (2007). GRA was performed using statistical analysis system software (SAS 10.0 version) and was used for obtaining the grey relational order (GRO) [12], which showed the correlations of BMI with WC, HC, WHR, and WHtR.

The original data have been listed below (Table 1 and 2). With the basic steps of GRA, the grey relational grades (GRG) were obtained (Table 3 and 4). The basic steps and formulas are described below:

\begin{tabular}{|c|c|c|c|c|c|c|c|}
\hline Gender & Age (yr) & Num. & $\mathrm{BMI}\left(\mathrm{x}_{0}\right)$ & WC $\left(x_{1}\right)$ & $\mathrm{HC}\left(\mathrm{x}_{2}\right)$ & WHR $\left(x_{3}\right)$ & WHtR $\left(x_{4}\right)$ \\
\hline \multirow{12}{*}{ Male } & 7 & 14 & $19.492 \pm 2.147$ & $56.386 \pm 5.407$ & $67.357 \pm 6.091$ & $0.839 \pm 0.054$ & $0.464 \pm 0.036$ \\
\hline & 8 & 23 & $20.264 \pm 2.287$ & $60.270 \pm 7.790$ & $71.391 \pm 8.427$ & $0.845 \pm 0.064$ & $0.470 \pm 0.055$ \\
\hline & 9 & 31 & $21.443 \pm 3.788$ & $66.326 \pm 9.149$ & $76.345 \pm 6.450$ & $0.868 \pm 0.077$ & $0.495 \pm 0.060$ \\
\hline & 10 & 32 & $22.624 \pm 2.636$ & $73.030 \pm 8.165$ & $81.084 \pm 6.176$ & $0.900 \pm 0.057$ & $0.525 \pm 0.052$ \\
\hline & 11 & 40 & $22.957 \pm 2.152$ & $74.108 \pm 8.249$ & $83.693 \pm 6.422$ & $0.884 \pm 0.050$ & $0.510 \pm 0.046$ \\
\hline & 12 & 63 & $23.791 \pm 2.202$ & $75.463 \pm 7.911$ & $86.563 \pm 5.499$ & $0.871 \pm 0.063$ & $0.500 \pm 0.050$ \\
\hline & 13 & 33 & $24.698 \pm 2.060$ & $78.997 \pm 7.330$ & $90.155 \pm 6.432$ & $0.876 \pm 0.050$ & $0.497 \pm 0.041$ \\
\hline & 14 & 20 & $24.753 \pm 2.261$ & $77.170 \pm 6.828$ & $92.105 \pm 7.065$ & $0.839 \pm 0.052$ & $0.474 \pm 0.037$ \\
\hline & 15 & 14 & $25.269 \pm 2.161$ & $76.107 \pm 7.236$ & $94.814 \pm 6.338$ & $0.802 \pm 0.044$ & $0.453 \pm 0.040$ \\
\hline & 16 & 22 & $26.109 \pm 2.605$ & $80.900 \pm 7.345$ & $94.809 \pm 5.264$ & $0.853 \pm 0.064$ & $0.492 \pm 0.050$ \\
\hline & 17 & 14 & $25.462 \pm 1.053$ & $78.936 \pm 4.114$ & $96.857 \pm 4.691$ & $0.816 \pm 0.037$ & $0.464 \pm 0.028$ \\
\hline & 18 & 17 & $27.009 \pm 2.514$ & $84.553 \pm 7.523$ & $99.476 \pm 5.959$ & $0.849 \pm 0.046$ & $0.497 \pm 0.041$ \\
\hline
\end{tabular}

Note: BMI: Body Mass Index; WC: Waist Circumference; HC: Hip Circumference; WHR: Waist-to-Hip Ratio; WHtR: Waist-to-Height Ratio

Table 1: Raw data for obesity indicators in male children (Mean \pm SD) 


\begin{tabular}{|c|c|c|c|c|c|c|c|}
\hline Gender & Age (yr) & Num. & $\mathrm{BMI}\left(\mathrm{x}_{0}\right)$ & $\mathrm{WC}\left(\mathrm{x}_{1}\right)$ & $\mathrm{HC}\left(\mathrm{x}_{2}\right)$ & WHR $\left(x_{3}\right)$ & WHtR $\left(\mathrm{x}_{4}\right)$ \\
\hline \multirow{12}{*}{ Female } & 7 & 5 & $19.883 \pm 1.760$ & $56.840 \pm 6.029$ & $64.260 \pm 9.113$ & $0.890 \pm 0.078$ & $0.473 \pm 0.038$ \\
\hline & 8 & 17 & $20.277 \pm 1.923$ & $58.306 \pm 6.890$ & $70.718 \pm 7.931$ & $0.825 \pm 0.037$ & $0.467 \pm 0.040$ \\
\hline & 9 & 10 & $21.430 \pm 1.921$ & $65.690 \pm 5.149$ & $78.690 \pm 4.196$ & $0.834 \pm 0.038$ & $0.497 \pm 0.036$ \\
\hline & 10 & 22 & $22.294 \pm 2.580$ & $67.473 \pm 9.225$ & $81.218 \pm 9.906$ & $0.831 \pm 0.066$ & $0.485 \pm 0.059$ \\
\hline & 11 & 13 & $23.895 \pm 3.426$ & $70.792 \pm 7.111$ & $85.231 \pm 6.013$ & $0.830 \pm 0.044$ & $0.491 \pm 0.050$ \\
\hline & 12 & 32 & $23.784 \pm 2.366$ & $70.466 \pm 6.789$ & $88.581 \pm 6.912$ & $0.796 \pm 0.056$ & $0.465 \pm 0.048$ \\
\hline & 13 & 27 & $25.107 \pm 3.652$ & $71.352 \pm 6.662$ & $90.581 \pm 6.107$ & $0.788 \pm 0.051$ & $0.469 \pm 0.038$ \\
\hline & 14 & 17 & $25.419 \pm 2.261$ & $72.771 \pm 7.346$ & $91.418 \pm 4.870$ & $0.796 \pm 0.066$ & $0.472 \pm 0.044$ \\
\hline & 15 & 6 & $26.742 \pm 2.766$ & $78.833 \pm 7.195$ & $96.167 \pm 6.765$ & $0.819 \pm 0.035$ & $0.503 \pm 0.041$ \\
\hline & 16 & 18 & $25.303 \pm 1.784$ & $77.167 \pm 6.224$ & $96.056 \pm 6.226$ & $0.804 \pm 0.058$ & $0.491 \pm 0.036$ \\
\hline & 17 & 15 & $25.519 \pm 1.108$ & $73.400 \pm 4.205$ & $94.800 \pm 3.802$ & $0.774 \pm 0.029$ & $0.481 \pm 0.025$ \\
\hline & 18 & 6 & $26.533 \pm 1.760$ & $76.167 \pm 7.935$ & $94.167 \pm 3.869$ & $0.807 \pm 0.056$ & $0.500 \pm 0.038$ \\
\hline
\end{tabular}

Note: BMI: Body Mass Index; WC: Waist Circumference; HC: Hip Circumference; WHR: Waist-to-Hip Ratio; WHtR: Waist-to-Height Ratio Table 2: Raw data for obesity indicators in female children (Mean \pm SD)

\begin{tabular}{|c|c|c|c|c|}
\hline Age $(\mathbf{y r})$ & WC & HC & WHR & WHtR \\
\hline 7 & $0.874 \pm 0.106$ & $0.818 \pm 0.136$ & $0.739 \pm 0.201$ & $0.819 \pm 0.107$ \\
\hline 8 & $0.732 \pm 0.148$ & $0.755 \pm 0.162$ & $0.735 \pm 0.157$ & $0.743 \pm 0.142$ \\
\hline 9 & $0.869 \pm 0.132$ & $0.879 \pm 0.119$ & $0.846 \pm 0.125$ & $0.873 \pm 0.131$ \\
\hline 10 & $0.787 \pm 0.160$ & $0.791 \pm 0.140$ & $0.725 \pm 0.159$ & $0.773 \pm 0.147$ \\
\hline 11 & $0.842 \pm 0.112$ & $0.857 \pm 0.101$ & $0.823 \pm 0.100$ & $0.853 \pm 0.097$ \\
\hline 12 & $0.726 \pm 0.153$ & $0.746 \pm 0.147$ & $0.741 \pm 0.165$ & $0.743 \pm 0.153$ \\
\hline 13 & $0.754 \pm 0.134$ & $0.795 \pm 0.126$ & $0.683 \pm 0.157$ & $0.720 \pm 0.136$ \\
\hline 14 & $0.827 \pm 0.134$ & $0.796 \pm 0.119$ & $0.750 \pm 0.167$ & $0.769 \pm 0.128$ \\
\hline 15 & $0.665 \pm 0.106$ & $0.651 \pm 0.155$ & $0.611 \pm 0.156$ & $0.729 \pm 0.178$ \\
\hline 16 & $0.728 \pm 0.185$ & $0.689 \pm 0.120$ & $0.698 \pm 0.202$ & $0.716 \pm 0.157$ \\
\hline 17 & $0.793 \pm 0.145$ & $0.750 \pm 0.156$ & $0.713 \pm 0.149$ & $0.775 \pm 0.136$ \\
\hline 18 & $0.730 \pm 0.162$ & $0.734 \pm 0.134$ & $0.663 \pm 0.148$ & $0.746 \pm 0.168$ \\
\hline
\end{tabular}

Note: GRG: Grey Relational Grade; BMI: Body Mass Index; WC: Waist Circumference; HC: Hip Circumference; WHR: Waist-to-Hip Ratio; WHtR: Waist-to-Height Ratio Table 3: GRG between BMI and other obesity indicators (males)

\begin{tabular}{|c|c|c|c|c|}
\hline Age $(\mathbf{y r})$ & WC & HC & WHR & WHtR \\
\hline 7 & $0.594 \pm 0.120$ & $0.466 \pm 0.105$ & $0.642 \pm 0.079$ & $0.698 \pm 0.226$ \\
\hline 8 & $0.691 \pm 0.180$ & $0.713 \pm 0.176$ & $0.753 \pm 0.141$ & $0.717 \pm 0.165$ \\
\hline 9 & $0.766 \pm 0.141$ & $0.782 \pm 0.146$ & $0.725 \pm 0.131$ & $0.845 \pm 0.104$ \\
\hline 10 & $0.691 \pm 0.148$ & $0.756 \pm 0.153$ & $0.688 \pm 0.187$ & $0.653 \pm 0.154$ \\
\hline 11 & $0.823 \pm 0.171$ & $0.807 \pm 0.165$ & $0.744 \pm 0.151$ & $0.826 \pm 0.108$ \\
\hline 12 & $0.848 \pm 0.126$ & $0.848 \pm 0.119$ & $0.816 \pm 0.120$ & $0.840 \pm 0.132$ \\
\hline 13 & $0.865 \pm 0.141$ & $0.845 \pm 0.122$ & $0.875 \pm 0.122$ & $0.877 \pm 0.138$ \\
\hline 14 & $0.798 \pm 0.186$ & $0.808 \pm 0.148$ & $0.768 \pm 0.171$ & $0.801 \pm 0.179$ \\
\hline 15 & $0.618 \pm 0.209$ & $0.631 \pm 0.166$ & $0.618 \pm 0.174$ & $0.634 \pm 0.155$ \\
\hline 16 & $0.724 \pm 0.153$ & $0.796 \pm 0.159$ & $0.701 \pm 0.168$ & $0.729 \pm 0.126$ \\
\hline 17 & $0.628 \pm 0.174$ & $0.703 \pm 0.179$ & $0.656 \pm 0.202$ & $0.659 \pm 0.153$ \\
\hline 18 & $0.572 \pm 0.132$ & $0.684 \pm 0.198$ & $0.705 \pm 0.126$ & $0.775 \pm 0.134$ \\
\hline
\end{tabular}

Note: GRG: Grey Relational Grade; BMI: Body Mass Index; WC: Waist Circumference; HC: Hip Circumference; WHR: Waist-to-Hip Ratio; WHtR: Waist-to-Height Ratio

Table 4: GRG between BMI and other obesity indicators (females)

1. Determination of the mean: Let BMI $\left(\mathrm{x}_{0}\right)$ be the reference series, and WC $\left(\mathrm{x}_{1}\right), \mathrm{HC}\left(\mathrm{x}_{2}\right)$, WHR $\left(\mathrm{x}_{3}\right)$, WHtR $\left(\mathrm{x}_{4}\right)$ be the comparative series. $\mathrm{X}_{\mathrm{i}}$ is named as the data series $(\mathrm{i}=0,1,2,3,4)$. Mean measurement is used to obtain the dis-dimension series. Here, the mean formula is as follows:

$$
x_{i}^{\prime}(k)=\frac{x_{i}(k)}{\frac{1}{n} \sum_{k=1}^{n} x_{i}(k)}
$$

$\mathrm{k}=1,2 \ldots \mathrm{n}(\mathrm{n}=5-63) ; \mathrm{k}$ is the case number. 
$\mathrm{x}_{\mathrm{i}}(\mathrm{k})$ and $\mathrm{x}_{\mathrm{i}}^{\prime}(\mathrm{k})$ are the values before and after treatment, respectively.

2. Identification of a difference series

The formula is . $\Delta i(k)=\left|x_{0}{ }_{0}(k)-x_{i}{ }^{\prime}(k)\right|$

Here, $\mathrm{i}=1,2,3,4$.

3. Casting of grey relational coefficients:

$$
\xi_{i}(k)=\frac{\min _{i} \min _{k} \Delta i(k)+\zeta \cdot \max _{i} \max _{k} \Delta i(k)}{\Delta i(k)+\zeta \cdot \max _{i} \max _{k} \Delta i(k)} \quad . \text { Here, } \mathrm{i}=1,2,3,4 .
$$

The discriminating coefficient $\zeta$ was taken as 0.5 .

4. Determination of the grey relational grade (GRG) and construction of the GRO

The GRG was calculated using the formula $\gamma_{i}=\frac{1}{n} \sum_{k=1}^{n} \xi_{i}(\boldsymbol{k})$ (Table 3 and 4 ).

Results

GRO can be obtained on the basis of GRG as given below [12].

The results for the obesity indicators for the males are given below.

$$
\begin{aligned}
& \text { Age 7: } \gamma\left(X_{\mathrm{WC}}\right)>\gamma\left(X_{\mathrm{WHtR}}\right)>\gamma\left(X_{\mathrm{HC}}\right)>\gamma\left(X_{\mathrm{WHR}}\right) \\
& \text { Age 8: } \gamma\left(X_{\mathrm{HC}}\right)>\gamma\left(X_{\mathrm{WHtR}}\right)>\gamma\left(X_{\mathrm{WHR}}\right)>\gamma\left(X_{\mathrm{WC}}\right) \\
& \text { Age 9: } \gamma\left(X_{\mathrm{HC}}\right)>\gamma\left(X_{\mathrm{WHtR}}\right)>\gamma\left(X_{\mathrm{WC}}\right)>\gamma\left(X_{\mathrm{WHR}}\right) \\
& \text { Age 10: } \gamma\left(X_{\mathrm{HC}}\right)>\gamma\left(X_{\mathrm{WC}}\right)>\gamma\left(X_{\mathrm{WHtR}}\right)>\gamma\left(X_{\mathrm{WHR}}\right) \\
& \text { Age 11: } \gamma\left(X_{\mathrm{HC}}\right)>\gamma\left(X_{\mathrm{WHtR}}\right)>\gamma\left(X_{\mathrm{WC}}\right)>\gamma\left(X_{\mathrm{WHR}}\right) \\
& \text { Age 12: } \gamma\left(X_{\mathrm{HC}}\right)>\gamma\left(X_{\mathrm{WHtR}}\right)>\gamma\left(X_{\mathrm{WHR}}\right)>\gamma\left(X_{\mathrm{WC}}\right) \\
& \text { Age 13: } \gamma\left(X_{\mathrm{HC}}\right)>\gamma\left(X_{\mathrm{WC}}\right)>\gamma\left(X_{\mathrm{WHtR}}\right)>\gamma\left(X_{\mathrm{WHR}}\right) \\
& \text { Age 14: } \gamma\left(X_{\mathrm{WC}}\right)>\gamma\left(X_{\mathrm{HC}}\right)>\gamma\left(X_{\mathrm{WHtR}}\right)>\gamma\left(X_{\mathrm{WHR}}\right) \\
& \text { Age 15: } \gamma\left(X_{\mathrm{WHR}}\right)>\gamma\left(X_{\mathrm{WC}}\right)>\gamma\left(X_{\mathrm{HC}}\right)>\gamma\left(X_{\mathrm{WHR}}\right) \\
& \text { Age 16: } \gamma\left(X_{\mathrm{WC}}\right)>\gamma\left(X_{\mathrm{WHtR}}\right)>\gamma\left(X_{\mathrm{WHR}}\right)>\gamma\left(X_{\mathrm{HC}}\right) \\
& \text { Age 17: } \gamma\left(X_{\mathrm{WC}}\right)>\gamma\left(X_{\mathrm{WHtR}}\right)>\gamma\left(X_{\mathrm{HC}}\right)>\gamma\left(X_{\mathrm{WHR}}\right) \\
& \text { Age 18: } \gamma\left(X_{\mathrm{WHtR}}\right)>\gamma\left(X_{\mathrm{HC}}\right)>\gamma\left(X_{\mathrm{WC}}\right)>\gamma\left(X_{\mathrm{WHR}}\right)
\end{aligned}
$$

The results of the physical examination (females):

Age 7: $\gamma\left(X_{\mathrm{WHtR}}\right)>\gamma\left(X_{\mathrm{WHR}}\right)>\gamma\left(X_{\mathrm{WC}}\right)>\gamma\left(X_{\mathrm{HC}}\right)$

Age 8: $\gamma\left(X_{\mathrm{WHR}}\right)>\gamma\left(X_{\mathrm{WHtR}}\right)>\gamma\left(X_{\mathrm{HC}}\right)>\gamma\left(X_{\mathrm{WC}}\right)$

Age 9: $\gamma\left(X_{\mathrm{WHtR}}\right)>\gamma\left(X_{\mathrm{HC}}\right)>\gamma\left(X_{\mathrm{WC}}\right)>\gamma\left(X_{\mathrm{WHR}}\right)$

Age 10: $\gamma\left(X_{\mathrm{HC}}\right)>\gamma\left(X_{\mathrm{WC}}\right)>\gamma\left(X_{\mathrm{WHR}}\right)>\gamma\left(X_{\mathrm{WHtR}}\right)$

Age 11: $\gamma\left(X_{\mathrm{WHtR}}\right)>\gamma\left(X_{\mathrm{WC}}\right)>\gamma\left(X_{\mathrm{HC}}\right)>\gamma\left(X_{\mathrm{WHR}}\right)$ 


$$
\begin{aligned}
& \text { Age 12: } \gamma\left(X_{\mathrm{WC}}\right)>\gamma\left(X_{\mathrm{HC}}\right)>\gamma\left(X_{\mathrm{WHtR}}\right)>\gamma\left(X_{\mathrm{WHR}}\right) \\
& \text { Age 13: } \gamma\left(X_{\mathrm{WHtR}}\right)>\gamma\left(X_{\mathrm{WHR}}\right)>\gamma\left(X_{\mathrm{WC}}\right)>\gamma\left(X_{\mathrm{HC}}\right) \\
& \text { Age 14: } \gamma\left(X_{\mathrm{HC}}\right)>\gamma\left(X_{\mathrm{WHtR}}\right)>\gamma\left(X_{\mathrm{WC}}\right)>\gamma\left(X_{\mathrm{WHR}}\right) \\
& \text { Age 15: } \gamma\left(X_{\mathrm{WHtR}}\right)>\gamma\left(X_{\mathrm{HC}}\right)>\gamma\left(X_{\mathrm{WC}}\right)>\gamma\left(X_{\mathrm{WHR}}\right) \\
& \text { Age 16: } \gamma\left(X_{\mathrm{HC}}\right)>\gamma\left(X_{\mathrm{WHtR}}\right)>\gamma\left(X_{\mathrm{WC}}\right)>\gamma\left(X_{\mathrm{WHR}}\right) \\
& \text { Age 17: } \gamma\left(X_{\mathrm{HC}}\right)>\gamma\left(X_{\mathrm{WHtR}}\right)>\gamma\left(X_{\mathrm{WHR}}\right)>\gamma\left(X_{\mathrm{WC}}\right) \\
& \text { Age 18: } \gamma\left(X_{\mathrm{WHtR}}\right)>\gamma\left(X_{\mathrm{WHR}}\right)>\gamma\left(X_{\mathrm{HC}}\right)>\gamma\left(X_{\mathrm{WC}}\right)
\end{aligned}
$$

\section{Discussion}

According to the data from the Chinese national surveys on the health, the prevalence of overweigh and obesity in children aged 7-18 years has increased 28 times since 2000 [14]. In the USA, compared to the hospital costs associated with type 2 diabetes, cardiovascular diseases, sleep apnea and orthopedic problems, costs associated with obesity have reached as high as \$127 million in the case of children [15]. Thus an increasing number of new measurements are being used for evaluating obesity. The researches indicated that WHtR, which is related to fat distribution, is a more efficient predictor of mortality than WC or BMI $[5,16]$.

A system review of children obesity in China showed that the definitions of childhood obesity used in Chinese studies varied. And recommended the use of gender-specific and age-specific BMI cutoffs when diagnosing obesity in Chinese children [17]. Therefore, we identified subjects based on the BMI cutoffs set by CDC.

$\mathrm{HC}$, WHtR, and WC may be used as alternative parameters for measuring obesity, with considerations of age and gender. In our study, the GRA for males showed that the BMI scores were strongly correlated with the HC for children younger than 14 years, but were weakly correlated with that for the children older than 14 years. In addition, BMI was closely associated with WHtR and WC, but the association between BMI and WHR was poor. However, in females, distinct association regularities were not observed among BMI, WC, HC, WHR, and WHtR. Generally, a strong correlation between WC, HC and BMI were found in females older than 9 years, but a weaker correlation was found between WC and BMI in children older than 14 years. These correlations between BMI and the WHtR and WC indicated that obesity mainly presents as abdominal obesity in children. However, nowadays, there are limited studies on the relationship between BMI and WC, HC, WHR and WHtR in children. BMI has been recommended worldwide as a standard criterion for defining obesity. However, the measurements of WC, HC, WHR, and WHtR are comparable to BMI to predict the risk of obesity related disorders such as type 2 diabetes [18] and possibly are superior to BMI as predictors of cardiovascular disease in both men and women [19]. A meta-analysis provides evidence that supports the superiority of measures of centralized obesity, especially WHtR, over BMI, for detecting cardiovascular risk factors in both men and women [5].

Although no exact correlation between BMI and other parameters, especially in females, was found in this study. Nevertheless, since children are at their peak growth stage, the WC, HC, WHR and WHtR should be defined according to age and gender as well.

Our study has some limitations. First, our sample size is relatively small in the obesity group. Second, the number of females analyzed was limited because the prevalence of obesity in males was higher than that in females. This may affect the result of our analysis for females.

\section{Conclusion}

WC, HC, WHtR, and WHR have different degrees of correlation with the BMI scores in children of different ages and gender, which revealed that they could be alternative parameters used for evaluating obesity BMI mainly, indicates the nutritional status of children and adolescents, while WC, HC, WHR and WHtR are more associated with body habitus, mainly with abdominal obesity. To accurately and completely estimate obesity in children, we need to consider multiple parameters such as WC, HC, WHtR, WHR, and not just BMI. Our study will provide some insights into the obesity study of children.

\section{Acknowledgement}

We thank the students and their parents in this study and thank the volunteers for assisting the survey. We also thank the Bureau of Education of Shantou, Guangdong province for organizing the physical examination. 


\section{References}

1. Jafar TH, Chaturvedi N, Pappas G (2006) Prevalence of overweight and obesity and their association with hypertension and diabetes mellitus in an Indo-Asian population. CMAJ 175: 1071-7.

2. Ng M, Fleming T, Robinson M, Thomson B, Graetz N, et al. (2014) Global, regional, and national prevalence of overweight and obesity in children and adults during 1980-2013: a systematic analysis for the Global Burden of Disease Study 2013. Lancet 384: 766-81.

3. Young JB (2010) Diabetes, obesity, and heart failure: the new pandemic. Methodist Debakey Cardiovasc J 6: 20-6.

4. Urbina EM, Kimball TR, Khoury PR, Daniels SR, Dolan LM, et al. (2010) Increased arterial stiffness is found in adolescents with obesity or obesity-related type 2 diabetes mellitus. J Hypertens 28: 1692-8.

5. Schneider HJ, Friedrich N, Klotsche J, Pieper L, Nauck M, et al. (2010) The Predictive Value of Different Measures of Obesity for Incident Cardiovascular Events and Mortality. J Clin Endocrinol Metab 95: 1777-85.

6. Seidell JC, Flegal KM (1997) Assessing obesity: classification and Epidemiology. Br Med Bull 53: 238-52.

7. Ketel IJ, Volman MN, Seidell JC, Stehouwer CD, Twisk JW, et al. (2007) Superiority of skinfold measurements and waist over waist-to-hip ratio for determination of body fat distribution in a population-based cohort of Caucasian Dutch adults. Eur J Endocrinol 156: 655-61.

8. Kelishadi R, Zemel MB, Hashemipour M, Hosseini M, Mohammadifard N, et al. (2009) Can a dairy-rich diet be effective in long-term weight control of young children? J Am Coll Nutr 28: 601-10.

9. Morrison JA, Glueck CJ, Daniels S, Wang P (2010) Determinants of persistent obesity and hyperinsulinemia in a biracial cohort: a 15-year prospective study of schoolgirls. J Pediatr 157: 559-65.

10. Molenaar EA, van Ameijden EJ, Vergouwe Y, Grobbee DE, Numans ME (2010) Effect of nutritional counselling and nutritional plus exercise counselling in overweight adults: a randomized trial in multidisciplinary primary care practice. Fam Pract 27: 143-50.

11. Koukoulis GN, Sakka C, Katsaros F, Goutou M, Tsirona S, et al. (2010) High rates of obesity prevalence in adults living in Central Greece: Data from the ARGOS Study. Hormones (Athens) 9: 253-62.

12. Deng Julong (2005) The primary methods of grey system theory. Huazhong University of Science and Technology.

13. Kuczmarski RJ, Ogden CL, Grummer-Strawn LM, Flegal KM, Guo SS, et al (2000) CDC growth charts: United States. Adv Data 1-27.

14. Wu Y (2006) Overweight and obesity in China. BMJ 333: 362-3.

15. Wang G, Dietz WH (2002) Economic burden of obesity in youths aged 6 to 17 years: 1979-1999. Pediatrics 109: E81-1.

16. Price GM, Uauy R, Breeze E, Bulpitt CJ, Fletcher AE (2006) Weight, shape, and mortality risk in older persons: elevated waist-hip ratio, not high body mass index, is associated with a greater risk of death. Am J Clin Nutr 84: 449-60.

17. Chen S, Binns CW, Zhang Y (2012) The importance of definition in diagnosing obesity: a review of studies of children in China. Asia Pac J Public Health 24 : 248-62.

18. Vazquez G, Duval S, Jacobs DR, Silventoinen K (2007) Comparison of body mass index, waist circumference, and waist/hip ratio in predicting incident diabetes: a meta-analysis. Epidemiol Rev 29: 115-28.

19. Lee CM, Huxley RR, Wildman RP, Woodward M (2008) Indices of abdominal obesity are better discriminators of cardiovascular risk factors than BMI: a metaanalysis. J Clin Epidemiol 61: 646-53.

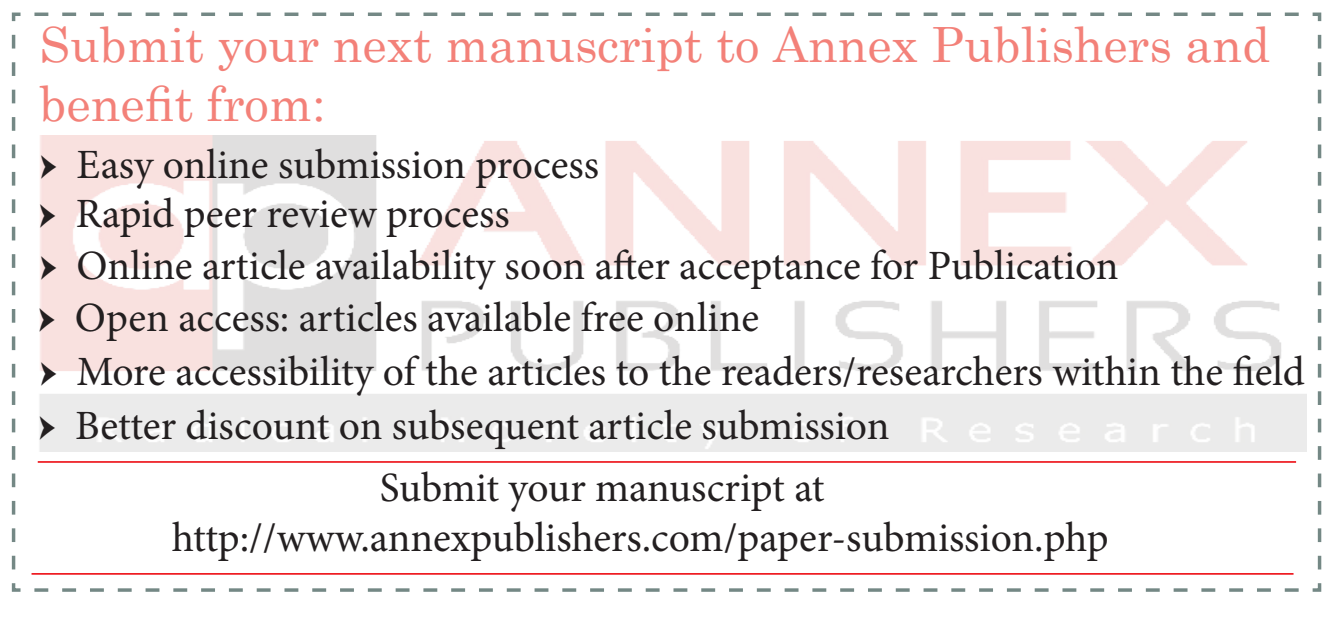

